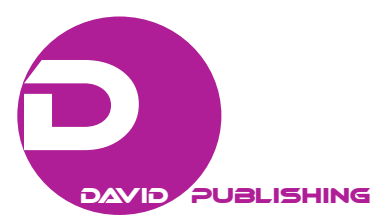

\title{
Temporal Disaggregation of Time Series Revisited
}

\author{
Erin M. Hodgess \\ Western Governors University, Salt Lake City, USA \\ Kendra Mhoon \\ University of Houston-Downtown, Houston, USA
}

\begin{abstract}
With the benefits of increased computing power and much improved software, temporal disaggregation is examined. Disaggregation, the process of obtaining high frequency data from low frequency data has been discussed for many years. This study examines three methods which utilize the autoregressive integrated moving average (ARIMA) model in a simulation study comparing parameter estimation, disaggregation mean square error, and forecast mean square error. Finally, the three methods are applied to a real-world time series.
\end{abstract}

Keywords: disaggregation, aggregation, ARIMA

\section{A Brief History of Disaggregation}

We start with the following notation: $x_{t}$ is the disaggregate series, $Y_{T}$ is the aggregate series, and $m$ is the order of aggregation. There are $n m$ total observations, with $n$ aggregate observations.

$$
Y_{T}=\sum_{t=m(T-1)+1}^{m T} x_{t}
$$

Equation 1 can be written as:

$$
Y_{T}=\left(1+B+\cdots B^{(m-1)}\right) x_{m T},
$$

where $B^{j} x_{t}=x_{t j}$. The actual process of disaggregation is the calculation of high frequency data from low frequency data; for example, converting quarterly data to monthly data. This process can be completed solely from the original series, or use multiple series in order to produce the final high frequency result.

The seminal disaggregation literature (Lisman \& Sandee, 1964) considered disaggregation of quarterly series. Lisman and Sandee's (1964) method of calculation lost the first and last observation of aggregate data. Boot, Feibes, and Lisman (1967) solved the loss of data issue with a first and second difference method for quarterly data. With their technique, only the first or second data points were lost. Finally, Cohen, Muller, and Padberg (1971) generalized the Boot method to all orders $m$ of aggregation, rather than simply quarterly to monthly. This generalization of the Boot method can be written as:

Erin M. Hodgess, Ph.D., course instructor, General Education Mathematics, Western Governors University, Salt Lake City, USA.

Kendra Mhoon, Ph.D., associate professor, Department of Mathematics and Statistics, University of Houston-Downtown, Houston, USA.

Correspondence concerning this article should be addressed to Erin M. Hodgess, 4001700 E \#700, Salt Lake City, UT, 84107, USA. 


$$
\begin{aligned}
L^{B 1} & =\sum_{t=2}^{n m}\left(\hat{x}_{t}-\hat{x}_{t-1}\right)^{2}-\sum_{T=1}^{n} \lambda_{T}\left(\sum_{t=m(T-1)+1}^{m T} \hat{x}_{t}-Y_{T}\right) \\
L^{B 2} & =\sum_{t=3}^{n m}\left(\Delta \hat{x}_{t}-\Delta \hat{x}_{t-1}\right)^{2}-\sum_{T=1}^{n} \lambda_{T}\left(\sum_{t=m(T-1)+1}^{m T} \hat{x}_{t}-Y_{T}\right)
\end{aligned}
$$

where $\Delta \hat{x}_{t}=\hat{x}_{t}-\hat{x}_{t-1}$.

\section{ARIMA Based Models}

\section{The Wei and Stram Method}

The properties of both aggregation and disaggregation have been investigated thoroughly in the following works: Stram and Wei (1986a; 1986b), Wei (1990), Wei and Stram (1988; 1990). Here is the basic process. An autoregressive integrated moving average (ARIMA) model is fit to the aggregate data. This is an $\operatorname{ARIMA}(p, d, r)$ model, where $p$ is the order of the autoregressive (AR) polynomial, $d$ is the order of integration, and $r$ is the order of the moving average (MA) polynomial. Thus we have:

$$
\alpha_{p}(\mathcal{B})(1-\mathcal{B})^{d} Y_{T}=\beta_{r}(\mathcal{B}) C_{T} .
$$

where $\alpha_{p}(\mathcal{B})=1-\alpha_{1} \mathcal{B}-\alpha_{2} \mathcal{B}^{2}-\cdots-\alpha_{p} \mathcal{B}^{p}, \mathcal{B}$ is the backshift operator such that $\mathcal{B}^{j} Y_{T}=Y_{T-j}$, $\beta_{r}(\mathcal{B})=1-\beta_{1} \mathcal{B}-\beta_{2} \mathcal{B}^{2}-\cdots-\beta_{r} \mathcal{B}^{r}$, and $C_{T}$ is a Gaussian white noise series with mean zero and constant variance $\quad{ }_{C}^{2}$. The AR and MA polynomials have no roots in common, and meet the criteria for stationarity and invertibility. In order to remove the differencing, we calculate

$$
U_{T}=(1-\mathcal{B})^{d} Y_{T} \text {. }
$$

The autocovariance matrix, $V_{U}$ is constructed based on the fitted values of $\alpha_{p}(\mathcal{B}), \beta_{r}(\mathcal{B})$, and $\sigma_{C}^{2}$. Next, we determine the order of the disaggregate polynomial. The order of the AR polynomial and the order of integration stay the same. However, the order of the disaggregate MA polynomial is calculated as (Wei \& Stram, 1990):

$$
q=p+d+1
$$

The maximum order of the MA polynomial.

The model of the disaggregate series will become:

$$
\phi_{p}(B)(1-B)^{d} x_{t}=\theta_{q}(B) a_{t} .
$$

The values of the AR polynomial become $\phi_{i}=\alpha_{i}^{\frac{1}{m}}$, where $m$ is the order of aggregation. Let

$$
w_{t}=(1-B) x_{t} \text {. }
$$

Then the aggregate autocovariances $\gamma_{U}$ are used to calculate the disaggregate autocovariances $\gamma_{w}$ to find the coefficients of the disaggregate MA polynomial via numerical methods. Hence the disaggregate differenced series will be: 


$$
w=\left(\hat{V}_{w}\left(C^{d}\right)^{\prime} V_{w}^{-1}\right) U
$$

The $C^{d}$ matrix is of the order $(n-d) \times(n m-d)$, and is constructed as:

$$
C^{d}=\left(\begin{array}{lll}
c & 0 & 0 \\
0 & c & 0 \\
0 & 0 & c
\end{array}\right)
$$

where $c$ consists of the coefficients of the polynomial $\left(1+B+B^{2}+\cdots B^{m-1}\right)^{d+1}$ and $U=C^{d} w$. The last step is to produce the final disaggregate series, without differencing:

$$
\hat{x}=\left(\begin{array}{cc} 
& \Delta_{n m}^{d} \\
0 & I_{d} \otimes J_{m}
\end{array}\right)^{-1}\left(\begin{array}{cc}
\hat{V}_{w}\left(C^{d}\right)^{\prime} V_{U}^{-1} \Delta_{n}^{d} \\
0 & I_{d}
\end{array}\right) \mathrm{Y} .
$$

$\Delta_{k}^{d}$ matrix is a $(k-d) \times k$ matrix where the $\delta_{i}$ are the coefficients of the expression $(B-1)^{d}$.

$$
\Delta_{k}^{d}=\left(\begin{array}{ccc}
\delta & \ldots & 0 \\
\vdots & \ddots & \vdots \\
0 & \cdots & \delta
\end{array}\right)
$$

The Boot methods are actually special cases of the Wei and Stram method, with the order of differencing at either 1 or 2, and the disaggregate autocovariance matrix, the identity matrix.

\section{Modifications to the Wei and Stram Method}

In Hodgess, the bounds for the MA polynomials were reduced. In one update, $q$ was set equal to $r$ and the autocovariances were updated accordingly. In the second approach, $q$ was set equal to 0 which resulted in an $\operatorname{ARI}(p, d)$ model. These approaches are data driven. These approaches showed some success in simulation studies, but were limited by computation power.

\section{Description of the Simulation Study}

An AR(1) series with $n m=360$ values was simulated. The order of aggregation was $m=3$. Values were summed to obtain an aggregate series with length $n=120$. In other words,

$$
\begin{aligned}
& x_{t}=\phi x_{t-1}+a_{t} \\
& Y_{T}=\sum_{t=m(T-1)+1}^{m T} x_{t}^{\text {and }}
\end{aligned}
$$

The values of $\phi$ ranged from -0.9 to 0.9 , in increments of 0.1 . The $a_{t}$ were random normal values from a standard normal distribution. The order of differencing was $d=0$. Thus $w=x$ and $U=Y$ gives

$$
\hat{x}=\left(\hat{V}_{w}\left(C^{0}\right)^{\prime} V_{U}^{-1}\right) Y .
$$

In Method 1, the best model was fit between an $\operatorname{AR}(1)$ and an $\operatorname{ARMA}(1,1)$ to the aggregate data producing a disaggregate $\mathrm{AR}(1)$ model, which in turn produces a disaggregate series. The second method fit an $\operatorname{AR}(1)$ 
model, utilizing an ARMA(1,1) model to produce the disaggregate series. Finally, the third method fit an AR(1) model and utilized an AR(1) disaggregate model to produce the final series. Note that Methods 2 and 3 have identical values for the AR(1) estimated parameter.

For each method, we found the estimated disaggregate $\hat{\phi}$, the disaggregate mean square error (DMSE), and the forecast mean square error (FMSE). We generated 12 extra data points in the initial set and compared these to the disaggregate forecast.

The formulas for the DMSE and the FMSE are:

$$
\begin{aligned}
D M S E & =\frac{\sum_{t=1}^{360}\left(x_{t}-\hat{x}_{t}\right)^{2}}{360} \text { and } \quad(17 \text { and 18) } \\
F M S E & =\frac{\sum_{f t=1}^{12}\left(x_{f t}-\hat{x}_{f t}\right)^{2}}{12}
\end{aligned}
$$

respectively.

Computing power and software has improved since the 1990s. Therefore, using 15,000 replications led to Tables 1, 2, and 3 .

Table 1

Parameter Estimation

\begin{tabular}{lll}
\hline$\phi$ & Method $1: \hat{\phi}_{1}(\mathrm{SE})$ & Methods 2 and 3: $\hat{\phi}_{2}, \hat{\phi}_{3}(\mathrm{SE})$ \\
\hline-0.9 & $-0.890(0.000)$ & $-0.893(0.000)$ \\
-0.8 & $-0.777(0.001)$ & $-0.797(0.000)$ \\
-0.7 & $-0.627(0.003)$ & $-0.708(0.000)$ \\
-0.6 & $-0.483(0.004)$ & $-0.625(0.001)$ \\
-0.5 & $-0.388(0.004)$ & $-0.539(0.001)$ \\
-0.4 & $-0.311(0.004)$ & $-0.444(0.002)$ \\
-0.3 & $-0.243(0.004)$ & $-0.343(0.003)$ \\
-0.2 & $-0.168(0.004)$ & $-0.238(0.003)$ \\
-0.1 & $-0.090(0.004)$ & $-0.125(0.003)$ \\
0.0 & $-0.003(0.004)$ & $0.001(0.003)$ \\
0.1 & $0.099(0.004)$ & $0.144(0.003)$ \\
0.2 & $0.202(0.004)$ & $0.296(0.003)$ \\
0.3 & $0.294(0.004)$ & $0.442(0.002)$ \\
0.4 & $0.361(0.004)$ & $0.556(0.001)$ \\
0.5 & $0.414(0.004)$ & $0.641(0.001)$ \\
0.6 & $0.509(0.004)$ & $0.714(0.000)$ \\
0.7 & $0.670(0.002)$ & $0.784(0.000)$ \\
0.8 & $0.797(0.001)$ & $0.855(0.000)$ \\
0.9 & $0.895(0.000)$ & $0.924(0.000)$ \\
\hline
\end{tabular}


Table 2

Disaggregation Mean Square Error

\begin{tabular}{|c|c|c|c|}
\hline$\phi$ & Method 1 DMSE & Method 2 DMSE & Method 3 DMSE \\
\hline \multirow[t]{2}{*}{-0.9} & 0.969 & 0.969 & 0.971 \\
\hline & 0.001 & 0.001 & 0.001 \\
\hline \multirow[t]{2}{*}{-0.8} & 1.015 & 1.018 & 1.040 \\
\hline & 0.001 & 0.001 & 0.001 \\
\hline \multirow[t]{2}{*}{-0.7} & 1.028 & 1.031 & 1.088 \\
\hline & 0.001 & 0.001 & 0.002 \\
\hline \multirow[t]{2}{*}{-0.6} & 1.011 & 1.012 & 1.072 \\
\hline & 0.001 & 0.001 & 0.002 \\
\hline \multirow[t]{2}{*}{-0.5} & 0.979 & 0.969 & 1.021 \\
\hline & 0.001 & 0.001 & 0.001 \\
\hline \multirow[t]{2}{*}{-0.4} & 0.939 & 0.914 & 0.970 \\
\hline & 0.001 & 0.001 & 0.001 \\
\hline \multirow[t]{2}{*}{-0.3} & 0.896 & 0.856 & 0.927 \\
\hline & 0.001 & 0.001 & 0.002 \\
\hline \multirow[t]{2}{*}{-0.2} & 0.851 & 0.800 & 0.888 \\
\hline & 0.001 & 0.001 & 0.002 \\
\hline \multirow[t]{2}{*}{-0.1} & 0.804 & 0.746 & 0.856 \\
\hline & 0.001 & 0.001 & 0.002 \\
\hline \multirow[t]{2}{*}{0.0} & 0.753 & 0.696 & 0.827 \\
\hline & 0.001 & 0.001 & 0.003 \\
\hline \multirow[t]{2}{*}{0.1} & 0.698 & 0.646 & 0.797 \\
\hline & 0.001 & 0.001 & 0.003 \\
\hline \multirow[t]{2}{*}{0.2} & 0.639 & 0.599 & 0.773 \\
\hline & 0.001 & 0.001 & 0.004 \\
\hline \multirow[t]{2}{*}{0.3} & 0.586 & 0.555 & 0.759 \\
\hline & 0.001 & 0.001 & 0.004 \\
\hline \multirow[t]{2}{*}{0.4} & 0.545 & 0.517 & 0.742 \\
\hline & 0.000 & 0.000 & 0.004 \\
\hline \multirow[t]{2}{*}{0.5} & 0.515 & 0.481 & 0.693 \\
\hline & 0.000 & 0.000 & 0.005 \\
\hline \multirow[t]{2}{*}{0.6} & 0.488 & 0.446 & 0.575 \\
\hline & 0.000 & 0.000 & 0.003 \\
\hline \multirow[t]{2}{*}{0.7} & 0.462 & 0.411 & 0.432 \\
\hline & 0.000 & 0.000 & 0.002 \\
\hline \multirow[t]{2}{*}{0.8} & 0.435 & 0.377 & 0.367 \\
\hline & 0.000 & 0.000 & 0.000 \\
\hline \multirow[t]{2}{*}{0.9} & 0.409 & 0.343 & 0.333 \\
\hline & 0.000 & 0.000 & 0.000 \\
\hline
\end{tabular}

Table 3

Forecast Mean Square Error

\begin{tabular}{llll}
\hline$\phi$ & Method 1 FMSE & Method 2 FMSE & Method 3 FMSE \\
\hline-0.9 & 2.700 & 2.695 & 2.707 \\
& 0.023 & 0.022 & 0.023 \\
-0.8 & 1.425 & 1.415 & 1.434 \\
\hline
\end{tabular}


Table 3 to be continued

\begin{tabular}{|c|c|c|c|}
\hline & 0.007 & 0.007 & 0.008 \\
\hline \multirow[t]{2}{*}{-0.7} & 1.182 & 1.171 & 1.202 \\
\hline & 0.005 & 0.004 & 0.005 \\
\hline \multirow[t]{2}{*}{-0.6} & 1.105 & 1.093 & 1.140 \\
\hline & 0.004 & 0.004 & 0.005 \\
\hline \multirow[t]{2}{*}{-0.5} & 1.072 & 1.062 & 1.124 \\
\hline & 0.004 & 0.004 & 0.005 \\
\hline \multirow[t]{2}{*}{-0.4} & 1.057 & 1.047 & 1.131 \\
\hline & 0.004 & 0.004 & 0.006 \\
\hline \multirow[t]{2}{*}{-0.3} & 1.049 & 1.041 & 1.146 \\
\hline & 0.004 & 0.004 & 0.006 \\
\hline \multirow[t]{2}{*}{-0.2} & 1.046 & 1.038 & 1.159 \\
\hline & 0.004 & 0.003 & 0.007 \\
\hline \multirow[t]{2}{*}{-0.1} & 1.044 & 1.037 & 1.172 \\
\hline & 0.004 & 0.003 & 0.008 \\
\hline \multirow[t]{2}{*}{0.0} & 1.043 & 1.037 & 1.199 \\
\hline & 0.004 & 0.003 & 0.009 \\
\hline \multirow[t]{2}{*}{0.1} & 1.043 & 1.040 & 1.216 \\
\hline & 0.004 & 0.003 & 0.009 \\
\hline \multirow[t]{2}{*}{0.2} & 1.046 & 1.045 & 1.235 \\
\hline & 0.004 & 0.004 & 0.010 \\
\hline \multirow[t]{2}{*}{0.3} & 1.055 & 1.057 & 1.251 \\
\hline & 0.004 & 0.004 & 0.009 \\
\hline \multirow[t]{2}{*}{0.4} & 1.075 & 1.078 & 1.239 \\
\hline & 0.004 & 0.004 & 0.008 \\
\hline \multirow[t]{2}{*}{0.5} & 1.111 & 1.111 & 1.219 \\
\hline & 0.004 & 0.004 & 0.007 \\
\hline \multirow[t]{2}{*}{0.6} & 1.178 & 1.183 & 1.218 \\
\hline & 0.004 & 0.004 & 0.006 \\
\hline \multirow[t]{2}{*}{0.7} & 1.324 & 1.330 & 1.295 \\
\hline & 0.006 & 0.006 & 0.006 \\
\hline \multirow[t]{2}{*}{0.8} & 1.721 & 1.733 & 1.605 \\
\hline & 0.010 & 0.010 & 0.009 \\
\hline \multirow[t]{2}{*}{0.9} & 3.432 & 3.455 & 3.046 \\
\hline & 0.031 & 0.032 & 0.027 \\
\hline
\end{tabular}

\section{Discussion of the Simulation Study}

For parameter estimation, the second and third methods (which are identical) seem to perform better on most of the values (Table 1). Yet Methods 2 and 3 tend to overstate the estimates in the initial positive values. The first method performs better for those values. In terms of DMSE, the second method outperforms the other two methods across almost all values (Table 2). Using the AR(1) model on the aggregate series and calculating the ARMA $(1,1)$ disaggregate model is very effective. Examining the first and third methods, which both have final AR(1) models, the first is the better of the two. Finally, the FMSE shows some unusual values. The second method does well throughout the negative parameter values, but then the first method dominates through most of the positive parameter values. The third method does not appear to be particularly fine-tuned for forecasting. 


\section{A Real World Example}

River water temperatures at the North Santiam River in Mehama, OR were gathered monthly data from January 2003 through December 2008. The data was aggregated into a quarterly series. For the first method, the $\operatorname{ARMA}(1,1)$ aggregate model was the best followed by an $\operatorname{AR}(1)$ disaggregate model. For Methods 2 and 3 , an $\operatorname{AR}(1)$ aggregate model was fit. For Method 2, the final disaggregate model was an $\operatorname{ARMA}(1,1)$, while Method 3 returned an AR(1) disaggregate model. Table 4 displays the final results.

Table 4

Santiam River Temperature

\begin{tabular}{llll}
\hline & Method 1 & Method 2 & Method 3 \\
\hline$\hat{\phi}$ & 0.9221 & 0.9699 & 0.9699 \\
DMSE & 0.3408 & 0.4905 & 1.1157 \\
\hline
\end{tabular}

The original parameter was $\phi=0.9799$. Methods 2 and 3 were very close in their estimations. In terms of the DMSE, Method 1 is most effective. These results show that all methods behave somewhat similarly and are all useful. Method choice should be based on the user-desired final model.

\section{Conclusion}

In the simulated and real-world data, no one method dominated all of the measures. That being said, the new methods showed great improvement over Wei and Stram (1990). These estimates are more consistent, based on the nearly negligible standard errors. Using these lower bound methods is an excellent way to handle disaggregation. For future work, bivariate time series in a similar study should be examined.

\section{References}

Boot, J., Feibes, W., \& Lisman, J. (1967). Further methods of derivation of quarterly figures from annual data. Applied Statistics, $16,67-75$.

Cohen, K., Muller, W., \& Padberg, M. (1971). Autoregressive approaches to the disaggregation of time series data. Applied Statistics, 20, 119-129.

Hodgess, E. (1995). Temporal disaggregation of time series. Philadelphia (PA): Temple University.

Lisman, J., \& Sandee, J. (1964). Derivation of quarterly figures from annual data. Applied Statistics, 13, 87-90.

Stram, D., \& Wei, W. (1986a). Temporal aggregation in the ARIMA process. Journal of Time Series Analysis, 7, $279-292$.

Stram, D., \& Wei, W. (1986b). A methodological note on the disaggregation of time series totals. Journal of Time Series Analysis, 7, 293-302.

Wei, W. (1990). Time series analysis: Univariate and multivariate methods. Redwood City, CA, USA: Addison Wesley.

Wei, W., \& Stram, D. (1988). An eigenvalue approach to the limiting behavior of the time series aggregates. Annals of the Institute of Statistics and Mathematics, 40, 101-110.

Wei, W., \& Stram, D. (1990). Disaggregation of time series models. Journal of the Royal Statistical Society, Series B, 3, $453-467$. 